\title{
The Emergency Public Relations Protocol: How to Work Effectively on Controversial Projects in an Academic Health Setting
}

\author{
B. R. Simon Rosser • Gunna Kilian • William G. West
}

Published online: 20 November 2012

(C) Springer Science+Business Media New York 2012

\begin{abstract}
Certain research topics-including studies of sexual behavior, substance use, and HIV risk-are more likely to be scrutinized by the media and groups opposed to this area of research. When studying topics that others might deem controversial, it is critical that researchers anticipate potential negative media events prior to their occurrence. By developing an Emergency Public Relations Protocol at the genesis of a study, researchers can identify and plan for events that might result in higher scrutiny. For each identified risk, a good protocol details procedures to enact before, during, and after a media event. This manuscript offers recommendations for developing a protocol based on both Situational Crisis Communication Theory and our experience as an HIV prevention research group who recently experienced such an event. The need to have procedures in place to monitor and address social media is highlighted.
\end{abstract}

Keywords Public relations $\cdot$ Media attack $\cdot$ Crisis communication $\cdot$ Social media

\footnotetext{
B. R. S. Rosser $(\bowtie) \cdot$ G. Kilian

HIV/STI Intervention and Prevention Program (HIPS),

Division of Epidemiology and Community Health,

University of Minnesota,

1300 South Second Street, \#300,

Minneapolis, MN 55454, USA

e-mail: rosser@umn.edu

\section{W. G. West}

Department of Writing Studies, College of Liberal Arts, University of Minnesota,

214 Nolte Center, 315 Pillsbury Dr SE,

Minneapolis, MN 55455, USA
}

There are many good things associated with doing cutting edge research, education, patient care, and/or community promotion in academia. However, the adage, "no pain, no gain" also applies. Cutting-edge research may be viewed as controversial by others. Sexual behavior and sexuality research, including HIV prevention education is one such "sensitive" area, where individuals and groups may organize to oppose the research. The media, advocacy groups, and congressional staffers may search funding websites looking for studies to oppose. Researchers can find themselves suddenly embroiled in situations where media inquiries demand answers to questions that bring academic research under intense political scrutiny. Whether the research is perceived to be conservative, liberal, or somewhere in between, and whether the inquiry comes as an exposé, community disquiet, an online petition, a congressional inquiry, professional criticisms by colleagues, or even death threats by those ardently opposed to the research, the reality is the same. The researchers suddenly find themselves in a highly emotional and potentially sensational media or political event, where the validity of the research, and sometimes the reputation of the scientists, is called into question.

In 2003, a list of National Institutes of Health (NIH)funded principal investigators circulated in Congress to have their funding rescinded. In 2011, a study that examined the effect a gay man's penis size has on his sex life- - backed by the National Institutes of Health - came under scrutiny by a group claiming the agency is wasting tax dollars at a time when the country is trying to control its debt. To increase the likelihood of an appropriate response, it is critical that researchers prepare an emergency public relations protocol prior to commencing a study. This manuscript offers recommendations for developing a protocol based on both situational crisis communication theory and our experience as an HIV prevention research group who recently 
experienced such an event. The need to have procedures to monitor and address social media is highlighted.

\section{Crisis Management Theory: A Brief Primer}

It is important to recognize that there is a science to crisis management. A signal event is the first appearance of a crisis (Kasperson et al. 2006). It may be an e-mail from a news organization asking for a comment or response or, even more innocuously, a posed question. The manifestation of this singular event is unknown but can result in a spectrum of outcomes ranging from insignificant (e.g., the news organization chooses not to run a story about the study) to the monumentous (e.g., multiple media sources run the story, resulting in heightened scrutiny of the study, the researchers and their institution, and the funding agency). At a signal event, the shape and size of the event to come is unknown, but the signal event can take us somewhat off our guard, and if a crisis does start, imagination can distort its importance.

An important first response is to understand and assess the size of the threat. Coombs's Situational Crisis Communication Theory (SCCT) posits that the strategic crisis response match the level of reputational threat and crisis responsibility (Coombs 2007; Coombs and Holladay 1996). SCCT is experimentally based and provides scientific evidence to guide decisions, particularly in the initially charged environment when "theory" seems to lack the certainty one seeks (Coombs and Holladay 1996, 2001; Kasperson et al. 2006). Coombs's theory is an outgrowth of attribution theory which underlies the type of response to a threat (Coombs and Holladay 2001; Kelley and Michela 1980). As shown in Table 1, SCCT helps define attribution of responsibility and prescribes an initial level of

Table 1 Situational crisis communication theory (SCCT): crisis response strategy guidelines

1. Informing and adjusting information alone can be enough when crises have minimal attributions of crisis responsibility, no history of similar crises, and a neutral or positive prior relationship and reputation.

2. Diminish crisis response strategies should be used for crises with minimal attributions of crisis responsibility coupled with a history of similar crises and/or negative prior relationship reputation.

3. Diminish crisis response strategies should be used for crises with low attributions of crisis responsibility, which have no history of similar crises, and a neutral or positive prior relationship reputation.

4. Rebuild crisis response strategies should be used for crises with low attributions of crisis responsibility (accident crises), coupled with a history of similar crises and/or negative prior relationship reputation.

5. Rebuild crisis response strategies should be used for crises with strong attributions of crisis responsibility (preventable crises) regardless of crisis history or prior relationship reputation.

6. Deny posture crisis response strategies should be used for rumor and challenge crises, when possible.

Source: Coombs (2007) response. When that is complete, the next step is to activate a response plan knowing which parts to activate first.

Crisis communication is a subspecialty of public relations used to describe an organization facing a crisis and the need to communicate about that crisis to stakeholders and the public. Typically, a crisis is an event that occurs unexpectedly, may not be in the organization's control, and may cause harm to the organization's good reputation or viability (Oak Ridge Associated Universities 2012). For example, a congressional inquiry about a study or a disease outbreak within a hospital is an unexpected event which threatens the organization's reputation if not handled adequately. Applied to media attacks on research, crisis communication defends a person or organization facing attacks on reputation.

There is common agreement among crisis communication experts that a plan be in place long before a crisis emerges (Coombs 2007; Covello 2003; Reynolds and Seeger 2005). Scholars may disagree about the level of detail needed in a plan, but few would advocate an improvisational response (Marra 1998). Key components of a protocol include a current contact list to activate a response team, designated spokespeople on behalf of the study, and designated staff responsibilities (Coombs and Holladay 1996). The most important element is a clear plan of response. Covello advocates for a thorough set of message maps (Covello 2003; Covello et al. 1998), which identify three key messages for any potential issue with three points of supporting evidence for each. Because complex research studies may raise multiple issues, we have used a broader approach which attempts to preemptively identify the concerns of those opposed to the research and to develop answers to address these concerns. Both approaches should result in two to three talking points per issue that address the key concerns.

Crisis communication can be counterintuitive. A natural response to an emerging crisis is to give too little or too much information. Identifying probable issues and refining a response prior to the crisis is a way of avoiding what Covello terms mental noise, a state that can reduce the ability to process information by up to $80 \%$ (Covello et al. 1998). A defensive position or tone by the principal investigator or agency can be self-defeating (Sandman 1993) since it allows others to define the terms of any discussion. To avoid this situation and identify probable issues that might come under attack, it is important to present any response as reasoned and open. To achieve this, it is critical to identify the audience for whom the issue is sensitive and the terms through which that audience sees the issue (Covello 2003). The audience-specific issues can be categorized into three types: overarching concerns, informational questions, or confrontational statements (Covello et al. 1998). Each type requires a slightly different response, but research indicates that $95 \%$ of these issues can be predicted and that key messages can be developed in advance for specific audiences (Covello 2012). 
The blueprint for risk communication success is anticipation, preparation, and practice (Covello 2012). What follows is a protocol developed for controversial research projects that provides the material necessary to manage a media inquiry, event, or crisis. It contains all the elements identified as essential for crisis communication.

This protocol is written in the belief that controversy is a necessary, even sometimes beneficial, part of our mission. As researchers, it behooves us to acknowledge this reality and to develop appropriate protocols to handle these situations. The senior author is an experienced NIH-funded scientist and Director of the HIV/STI Intervention \& Prevention Studies program, whose mission includes developing and testing "next generation" approaches to HIV prevention and care. Although the immediate context of this protocol is sex and sexuality research focused on developing and testing the effects of sexual health and HIV prevention programs for high-risk populations, we anticipate the protocol has broader application to scientists working in other areas of research as well.

\section{Writing an Emergency Public Relations Protocol}

\section{Starting a Controversial Project}

The key to successful handling of controversial situations is careful and adequate planning. When starting a project, the principal investigator often has demanding timelines for project implementation. Establishing equivalent timelines to handle the political realities of the project is just as important. Therefore, we recommend the following:

1. Create a list of key personnel in event of a media emergency. Identify the people you might need to draw on if you or your project becomes a media story. Identify the key persons in your organization and an alternate for each key organization. If you know your project is likely to be controversial, contact the public relations office at the relevant level(s); for example, the school, academic health center, university, or funding agency levels. They can assist by helping you prepare and handle any controversy. Collect the 24-h contact information for each key person, including both e-mail and phone contact (office, after hours, and mobile phone), then assemble the information into an email and text messaging emergency list. For example, for each of our studies, we have an emergency contact list that includes the following information:

(a) Contact information for the principal investigator, co-investigators, and, where relevant, key staff and consultants. (b) Contact information for the relevant public relations officers at the department, school, health center, and Office of the President levels.

(c) Contact information for a staff member in the institutional review board human subjects' protections (IRB) office.

(d) Contact information of the project officer of the funding agency.

(e) Contact information for anyone else deemed likely to be asked to comment or drawn into the controversy if the study was highlighted in the media. This may include public relations at the state health department, community-based organizations working in the area under study, and, potentially, community advisory board members.

(f) Contact information for state and federal representatives. Where a researcher has good relationships with their Congressional, Senate representatives, or local politicians, the study should consider either including the press officers of the supportive politicians on this list or creating a secondary list of key stakeholders to be kept informed of developments during a crisis.

The emergency contact list should be considered highly confidential since it contains personal information such as home and mobile phone numbers.

The purpose of this list is threefold. First, by having all the contact information in one place, the principal investigator has access to everybody $\mathrm{s} /$ he needs in an emergency. Second, the list facilitates communication between those involved without having to go through the principal investigator by sharing the list with everyone on it. Third, the list is a mechanism whereby everyone can be kept informed as an event is unfolding. Where a list has multiple people in a similar role (e.g., our protocol has public relations specialists at the school, health center, president's office, health department, and funding agency), we recommend identifying one person to function as the lead public relations advisor to the study, with responsibility to consult with the other public relations persons as needed.

2. Identify how your study is most vulnerable to attack or controversy. Develop message maps that address the following:

(a) Key messages or talking points to respond to any inquiry.

(b) Specific answers to the most difficult, embarrassing, and negative questions you could be asked about the project.

(c) Answers to supplemental questions that could arise out of an inquiry. During a crisis, you normally do 
not have time to develop answers, let alone get feedback on them.

One way to do this is to ask the team of investigators, staff, and/or community advisory board to role play or place themselves in the position of individuals at the organizations most opposed to the research. Most likely, this will include some combination of those who may be opposed to the research on fiscal grounds; those opposed to the research on some moral, ethical, or religious grounds; and/or those opposed because the study touches on other sensibilities (e.g., the target population feels vulnerable to exploitation or manipulation or some aspect of the intervention is a concern). Brainstorm the list of questions from the perspective of persons opposed to the research. The list of questions is critical since the whole media response is based upon them. The role play aspect is also important, as it can be helpful to frame the questions in the mindset of those antagonistic to the study; this is likely to be the language in which questions are framed. In Fig. 1, we provide an example of the types of questions we have developed on a recent study and sample answers to select questions.

Once the questions are identified, answers can be framed. Ideally, the answers should directly address the concern. For example, if the concern is economic (e.g., a "waste of taxpayer funds"), where there is a legitimate argument to be made about the research leading to economic savings for the taxpayer, these should be stated. Alternatively, where the economic savings are not known, the investigators can consider adding an economic analysis to address the question. Finally, review answers and identify key talking points.

3. Think through how best to protect your project. For example, community review boards and community advisory boards exist to assist investigators. If there is a concern that someone from the target population may object that the research is community-insensitive or culturally inappropriate, enlisting the help of members of the community can help the study avoid such pitfalls while adding community credibility. When researching illegal or stigmatized behavior, a Certificate of Confidentiality from the National Institutes of Health may protect data privacy. For educational initiatives, it may be helpful to submit any curricula to the relevant State Program Review Panel; in HIV prevention, such panels are authorized to assess whether an intervention is community appropriate and educational.

4. Consider briefing key allies. For research on controversial projects, especially where there may be concerns about economic waste, a key consideration is whether or not to brief local politicians (for statefunded research) and federal politicians (for federally funded studies) about the research. Most research universities have liaisons with their state and federal representatives who can advise on who among the representatives would most appreciate knowing about the research. In our research, we take the view that potential allies cannot defend our work if they are not aware of what it is about. By briefing a politician on the aims of the research and its benefits to the local community, politicians become mindful of the importance of the research and research funding to their constituency and potential allies.

5. Identify the overall response strategy. Meet with your team to agree on an overall public relations response strategy, including rules, expectations, and boundaries regarding media in case of a media situation. For each study, it is important to clarify the general strategy, which may change over the different phases of study. A general strategy should identify a decision on whether to take a low or high profile regarding media on this particular study. For example, for a phase III trial, you might decide to adopt a low profile, prioritizing completing the research over any publicity. But for a phase IV or V trial, where there is likely to be substantial community involvement in rolling out an intervention, a higher profile might be appropriate or necessary. The protocol should clearly state who is to liaise with the media, and staff should be trained in how to respond to media inquiries. The staff training should specify what information staff may or may not disclose to others about the research, including the use of social media during the attack.

6. Establish a "media emergency" file. This should be a one-stop place to access all the critical information needed if there was a public relations event, including a crisis or attack. In this file, place the following key information:

(a) Names, work phones, after-hours numbers, e-mail, and other contact information of your key persons. Key persons should include:

(i) All staff on the project.

(ii) Your project officer or other contacts at your funding agency/ies.

(iii) All persons who comprise your "emergency contact" list (see above). By grouping these people in one e-mail group, you can readily communicate in case of emergency.

(b) A copy of your key messages or talking points, questions and answers, and supplemental questions and answers. Whether electronic or in paper form, this should be clearly marked "CONFIDENTIAL, 
The Men's INTernet Study (MINTS) was one of the first studies of Internet-based HIV primary prevention. The objective was to develop and test a sexual health promotion program designed to lower the unsafe sexual behavior of men who use the Internet to seek sex with men. While the study was well justified by the epidemiology, and scientific need to discover ways of applying new technology to preventing HIV, from an ultra-conservative perspective, it raised concerns about the target population (gay men), and approach (sexually explicit content).

Our team identified the following questions as highly relevant from a conservative perspective:

\section{Are you promoting homosexuality?}

a. No. The program is designed to promote health and responsible sexual behavior, in order to prevent the spread of HIV and other STDs.

b. The new online intervention has been reviewed by the Minnesota Department of Health program review panel (the federally established review group to examine this issue). They concluded that the materials do not promote homosexuality or heterosexuality.

\section{Are you showing porn to gay men?}

No commercial pornography is used in this intervention and no unsafe sex is shown. We do show some sexually explicit images that were photographed by a photographer who has won international awards for his HIV prevention photography (used with permission). This intervention is based on the type of education we provide our medical students so they can be fully informed about sex and comfortable discussing concerns with their patients. Naturally, if you want students or groups at high risk to be informed, some of the material used in the seminar needs to be clear. It is simply more effective education to show people how to protect themselves, rather than just tell them.

Previous research which we have publishedon both the theory and the behavioral aa effects of this approach shows that it is effective at reducing risk, specifically among men who have sex with men. So we have a new approach to prevention which we know works offline, and now we are studying if it works online. If it does, this approach may save thousands of men from becoming infected.

\section{Can't you just tell them not to have sex?}

Scientists have compared abstinence based and just say no campaigns to educating people about sex and found they are simply not as successful. In February 1997, the NIH published a consensus statement on HIV preventionin whichthe nation's scientists concluded that only telling people to be abstinent does not work long term.

\section{But these are people infected with a deadly virus. Aren't you even concerned that} they may go and infect others precisely because of attending your program.

Every sexual transmission of HIV, by definition, involves a person infected with the virus. We know this and need to develop good programs to help people living with HIV. Just telling people not to have sex for the rest of their lives is not effective.

Fig. 1 Case study: The Men's INTernet Study (MINTS) 


\section{Can't you tell them that it is a sin?}

Our role is to promote health and healthy behaviors as faculty in a public research university.

\section{How do you know your programs (interventions) are not too explicit for your} population? Aren't you just feeding participant's prurient sexual interests?

That's why we are conducting this research. Until you test an intervention on the population it is designed for, you cannot know whether something is not explicit enough, just right, or too explicit.

\section{But "so-and-so"*said the images were pornographic and disgusting.}

In designing this intervention we asked over 2700 American high-risk men what was acceptable or not acceptable. Over $98 \%$ of men at greatest risk indicated the level of explicitness was appropriate for them. We tested the intervention in the lab and men at greatest risk said the images were fine (and one actually recommended we include more). As scientists we need to trust the information the people at risk are telling us. To be effective, the intervention needs to bereal, relevant, and acceptable to them. Otherwise, it's very unlikely that a program will work.

In the real world we're trying to stop one of the greatest killers on the face of this earth and the greatest killer in this population. Clearly noteveryone is going to agree about everything, but we're convinced that when $98 \%$ of high risk men state this is what is needed, we must try it.

\section{But "so-and-so"*said they were traumatized and never expected to see something so disgusting.}

The intervention is designed to target the men at greatest risk for HIV. To enrollin the study, men had to be on an adult sex site for MSM (which means that they have already seen similar images). Only men who have recently engaged in high risk sex could enter the study. Then, each man needed to indicate, not once but twice that they were comfortable viewing sexually explicit materials, otherwise they were thanked but excluded. Next, before starting, each person was invited to ask any questions of the researchers via email or telephone and if uncertain to discuss their concerns with us. In addition, if a subject saw anything they did not wish to see, because this is an Internet-based study, they could simply leave with one click of a button.

\section{Would it not have been better to just "tone down" the images?}

From our previous research, $95 \%$ of men and women (both homosexual and heterosexual) who participate in sexual health programs report that the sexually explicit media is helpful to their understanding of human sexuality. As previously noted we surveyed 2,716 men, $98 \%$ of whom state this is acceptable. In online interventions visual communication is even more necessary since people don't read lots of text. This isn't entertainment. This is an education program designed by public health professionals, computer scientists and HIV preventionists to stop Americans from beinginfected by HIV.

Fig. 1 (continued) 


\section{Why don't you have a program for heterosexuals with HIV?}

Although men who have sex with men constitute less then $5 \%$ of the American population, they are 22 times more at risk for HIV and make up over half of all new infections in the USA. They are also one of the first populations to use the Internet. If America is to be serious about stopping HIV, we need to focus on this population and risk through Internet liaisons. If we find that the interventions are as effective as we hope, then we plan to publish our results so we and others can develop similar programs for other populations.

\section{Why an Internet study?Won'tthis prevent those not online from participating for} example poor Men of Color?

This study is specifically looking at how we can use the Internet to stop the spread of HIV. We have other studies looking at offline ways of stopping HIV also. Because severa studies have shown Internet-liaisons as associated with the spread of HIV and other sexually transmitted infections particularly among Men who use the Internet to seek Sex with Men, it makes sense to design an online intervention for this population. After all, this is where they are meeting their partners.

\section{Why are you not more worried about children and others who might be offended} seeing this website by mistake?

We have strong computer security in place. Access to the site is possible only for subjects that are enrolled in the study. In addition, we have designed measures to ensure that the people in the study are really who they say they are. In Internet research, because you don't meet the person face-to-face, you can never be $100 \%$ certain. But even in the unlikely event that a minor was in an adult sex site, we think that getting them out of there to answer questions about risk, and to receive education is a lot better and lower risk than their staying at such a site.

\section{Why fund a study of only gay men when AIDS is decreasing in gay men and} increasing in other populations?

MSM are the largest single group becoming infected with HIV in the United States. Data and alerts from CDC suggest that prevention programs targeting this population may $b$ failing. So it made sense to us, to do the greatest good, to develop new and more effective programs for this population.

*So-and-so represents some unknown person who could be a participant who was involved in the research and was genuinely offended, someone who undertook the research in order to declare themselves offended, a third party who gained access to the study and had a negative reaction, a politician, or an expert with an opposing view.

Fig. 1 (continued) 
FINAL" and should be dated. Do not release this document to any beyond the key personnel.

(c) A summary of the project with a clear description of the information media may request:

(i) Abstract, project overview, brief description. For projects that are federally funded, it is helpful to use the same descriptions as on the application. For this reason, it is smart when writing the title and abstract for an application, to consider how they frame the study as the "publically accessible" parts of a research application. Some researchers may choose to avoid use of common terminology (e.g., "abortion," "gay/homosexual," "sex") in the title and abstract, if it makes the study easier to be detected through database searches. If the goals or other information have been amended since the award, then a revised up-to-date abstract, project overview and description are needed.

(ii) Governance, including the name and contact numbers for the IRB.

(iii) Funding, including the total amount of funding and the current funding.

(iv) Funding agency contact.

These four pieces of information should be in a form that can readily be emailed or faxed, if necessary, to any party, including the media. Together, they represent the publicly accessible information about a study in a form ready to be released.

(d) A copy of any key legislation relevant to the project as a reference.

(e) A copy of the human subjects' number and date authorized by the IRB.

(f) A copy of the Certificate of Confidentiality (if appropriate).

(g) Any media releases (positive or negative) about the project. It is important to have ready access to any past information the media or others may have.

7. Distribute the file to all key personnel. All key persons should receive an electronic copy of this file, marked "CONFIDENTIAL."

8. Contain the response to key personnel. Establish clear boundaries with all other persons on the project, including co-investigators, the project coordinator, research assistants, administrative assistants, and any others who may be solicited for information during a breaking news story. With community advisory boards, volunteers, and project employees, it is important to explicitly state at the start of the study the processes to deal with any disagreements. For example, internal disagreements are to be handled internally, either through the principal investigator, the study ombudsperson, or the principal investigator's immediate supervisor. All new hires are informed at orientation, that no employee is to go directly to the media, the public, or beyond the departmental head under any circumstances without first going through these channels. It is also important to ensure the protocol has the support of one's supervisors (e.g., department head). For smaller projects, we recommend the principal investigator be the only one who should speak on behalf of a study, as he/she is normally the best informed about the study, and consequently, the one most qualified to speak about it. A written plan should detail who is to handle media responses if the principal investigator is absent for some reason. For larger projects, the group may have a spokesperson designated for public relations, who may also be a university public relations liaison.

9. Be ethical, honest, and have integrity. Have an absolute commitment to conduct all aspects of your research or project ethically, honestly, and with integrity, and to convey that expectation to all staff members. In making decisions, consider how the decision would look in a negative light and, where possible, use co-investigators and peer faculty supervision to discuss controversial decisions prior to implementation. Create paper or electronic trails to protect your integrity and the nature of the research project. In particular, work closely with the IRB human subjects' committee, informing them of any negative outcomes or events as soon as possible. We take the approach that our IRB cannot support us if they are unaware of what has happened.

10. Be prepared. We treat our emergency public relations protocol in a similar way to IRB approval, meaning that we do not proceed with a study until the public relations protocol has been written and tailored for that study. Before any public aspect of the study launches (e.g., advertising and recruitment) and, preferably, long before any publicity is released about the study, we develop written responses to the most likely questions a study could be asked.

\section{During a Particular Incident}

We recommend doing these things in the following order:

1. Give yourself time. The greatest pressure in these situations usually is related to time. It can be helpful to remember that you do not need to "jump" immediately just because the media wants to air something or because someone wants something. The first goal is to claim your time, which may involve stalling the request. If the first inquiry is by phone, state, "It's not convenient 
just now, let me call you back in...."; if in person, "I need to attend to some things first; I can see you in..."; or if in public, "I think it's more appropriate that we discuss this elsewhere."

In our experience, there are likely to be two senses of time. When a journalist is initiating the story, there may be considerable flexibility as to when it is released. Hence, it is important to negotiate when the story will break in order to give yourself the time needed for a measured, accurate response. Alternatively, when a story is being pushed by a group opposed to the research, there may be very little flexibility with time. In this case, the most important decision is to identify what information to get to the media agency before the story breaks so that both sides of the issue can be presented. Hopefully, by carefully preparing the questions, responses, talking points, and abstract in written form ahead of any event, the principal investigator is prepared to respond quickly if necessary.

2. Activate your support system. A brief e-mail entitled "EMERGENCY PUBLIC RELATIONS PROTOCOL ACTIVATED" to all key persons is next. In the email body,

(a) explain the situation (event or crisis)

(b) ask key persons to activate their system (normally to inform the relevant persons at their level as to what is happening)

(c) attach the "media emergency" file to the email (so that everyone has ready access) and ask them to reread it if necessary

(d) describe what communication or next steps you need from them. For example, "Joe Smith just called to say Senator X has launched an inquiry into our study. Please do the following: Alert your respective heads so they are informed. Please reread our responses to common questions. I will call you when I have more information, and to seek your recommendations."

3. Keep good boundaries. It is a good idea, particularly when many media are calling, to remind all staff, especially new staff, of the boundaries of who is authorized to speak to the media. Unauthorized staff should be instructed:

(a) to state pleasantly but firmly who is the correct person to contact

(b) to offer to take a message, including a request for specific questions

(c) to alert you in person to any relevant communications

4. Use your support system. Having e-mailed the key persons, the next stage is to activate a response. It is critical for the principal investigator to recognize that there are experts at most institutions who are trained in how to deal with such events. Therefore, listening to and being guided by the public relations expert(s) is key. In our experience, since we had several public relations liaisons involved, it was most helpful to have one public relations person identified as the key advisor to the principal investigator. In addition to advising the principal investigator, this person's role includes both monitoring the media story, including any related blog and Twitter activity, as well as providing updates to the principal investigator or team on significant developments. This frees up the principal investigator to be responding to the event. The broader team can be kept informed as needed or drawn upon to address specific aspects. Keeping one's supervisor informed is also helpful as $\mathrm{s} / \mathrm{he}$ is probably the person who knows you best, can judge how you are responding, and can remind you of internal preferences.

Thus, the principal investigator and public relations liaison, aided by the wider response team as needed, discuss the proposed plan of response and agree on a response strategy. Throughout the controversy, we keep the feedback loop active by discussing intentions, progress, and other options throughout the inquiry. Because the controversy often changes quickly, it can be important to invite input, proactively, on what to do if the situation changes.

The decision on how to respond to each aspect of the media event should reflect the broader philosophy on addressing the media. For example, in the situation we recently faced in the Men's Internet Study (MINTS) study, colleagues who had been similarly attacked in previous years offered referrals to individuals in the media who were sympathetic to the research we were undertaking. We met as a team but decided we would not engage such media, since the overall strategy was to keep a low profile; anything more would only prolong the story. In this instance, the strategy was successful.

5. Use your key messages, questions, and answers sheet. Once the plan of response is confirmed, the next thing to do is to review the key talking points, and questions and answers. For phone interviews, we recommend placing these in front of you so you can read your response and focus on the key messages. For television and personal interviews, it is still important to read these before going into the interview. It helps to keep focused. Some general tips that usually apply:

(a) Respond only in writing whenever possible. For researchers untrained in media, this lowers the risk of an interview opening up new questions or offthe-cuff comments inflaming rather than diffusing the controversy.

(b) Adopt a pleasant, reasonable tone, both in written communication and in person. It defuses tension, you appear reasonable, and it gives you more time to respond. 
(c) View the situation as an opportunity, not an attack. The public has a right to know what we do with public funds. The media have the right and obligation to investigate. If you believe in your study and you have conducted it ethically, honesty, and with integrity, have confidence in your abilities. Whatever the question, bring the focus back to the key messages or talking points.

(d) Use normalizing language. "This is just a study... we're only looking at...much like other studies we are examining..."

(e) Do not attack the messengers; enlist them as allies. No matter how much we dislike the situation, the media are usually messengers who are only trying to investigate a story. Separating them mentally from the person/organization who may be challenging your work is important. In the media, avoid becoming defensive or passive-aggressive (e.g., not responding at all or attacking indiscriminately). Two helpful strategies are to answer all reasonable questions rationally and courteously and to give yourself the right not to answer unreasonable or inflammatory questions. Keep your cool.

(f) Contain the situation. Do not give out more information than asked for. Be precise, concise, and stick to the facts.

(g) Take measured steps to alleviate anxiety. One or two deep breaths before, during, and after interviews helps activate the parasympathetic division of the autonomic nervous system. It is important to look and feel as relaxed as possible while handling the anxiety associated with the situation.

(h) Critically distinguish between your needs and their needs. In the middle of a situation, you can receive all sorts of requests. Someone challenging your study may have a right to certain information; that does not make you their research assistant. If something is public information, you can direct them to the relevant agency rather than tell them that information.

(i) Facilitate information to allies (e.g., public relations staff, funding agencies, attorneys) and control information to all other interested parties. In the middle of a controversy, it can be helpful to not answer your phone and to rely instead on voicemail; to have someone else screen your calls, specifically asking what key questions the caller has and passing them on to you; and to schedule media interviews after the initial inquiry in order to give you time to prepare.

(j) Keep the team involved. You do not have to shoulder this alone. With the public relations liaison and key persons, seek input on what strategies they recommend for this particular situation. When key persons give you contradictory information (e.g., one person recommends speaking to the media and another to lie low), where possible alert the group to the different feedback you are receiving and ask them to come to consensus.

6. Keep the study on timeline. You cannot tell at the start or during a media event, how long the media attention will last. Having a person designated to address the eventusually the principal investigator and/or the public relations liaison-enables the rest of the team to remain focused on conducting the study. The co-investigators and project coordinator are included on the emergency protocol to keep them informed; but to maintain productivity, their primary role during an event is to ensure the study continues running smoothly. To help them stay focused, we normally do not enlist other staff to serve on the emergency public relations response list. Clearly, it is important to consider the impact of the media event on the science being conducted. Depending on the phase of study, it may be prudent to postpone some study milestones, such as launching recruitment, until the media event has subsided.

7. Practice self-care. Dependent on your personal style, self care can include:

(a) Grounding your feelings. Touch is the sense that best grounds most of us when we are anxious. Having a partner, colleague, or someone close by who can give you a hug or affirming touch can be important. Others may find physical activity helpful during ongoing crises. Still others may need solitude and time away from phones and computers to get their bearings.

(b) Personal support. It is important to take the time to call a supportive colleague or friend to talk to or accompany you to the situation.

(c) Distancing yourself from negative people. The middle of a crisis is not the time to solicit or receive negative feedback from colleagues. While constructive criticism may improve your skills, limit contact with people from whom you are likely to receive unhelpful negative feedback. It merely serves to lower your confidence. Tell such colleagues that you will be happy to discuss the situation after it has resolved.

(d) Articulating your needs. Think about what else you may need and who the appropriate person to respond to that need is. For instance, if you have been up all night fielding inquiries or worrying, talk to your supervisor about taking time off for sleep. Others cannot help if they do not know what happened. 


\section{After the Incident}

1. Inform key personnel of the outcome(s). Informing key personnel includes disseminating media reports (positive and negative) to keep them updated. Frequently, the list of key contact persons may evolve across an event. Ask all persons to update their files with the new information at the end of an incident. Do not forget to update your own file.

2. Review the protocol. What parts of the protocol worked well? What parts were unhelpful or need revision? If you need to adjust the protocol, meet with key personnel to revise the protocol.

3. Thank the personnel for their support and address their concerns. While you may have shouldered most of the work in responding to the crisis, a public relations event likely has had effects on other personnel. It is important to manage the concerns and consequences both during the event and after the event. A debriefing session that solicits and addresses staff concerns may be needed. For example, during the MINTS event, some doctoral students were worried about the implication of any problems of funding reduction on their job security and education. Addressing concerns and thanking staff for their support and help in responding to the event is necessary.

4. Learn. Each controversial situation is a learning situation if we take the time to reflect on the process and improve our skills. Only seeing it as an attack, and not adequately debriefing, prevents us from becoming more accomplished at handling future situations and, possibly, better researchers.

5. Identify who else needs to be informed about what happened. For example, if an incident was local, does this have national implications? Who else should be provided with a summary of the study so as to respond efficiently if there is another controversy?

6. Practice self-care. Most of us did not become faculty or researchers because we wanted to be attacked or profiled in the media. We can become angry that, in our field of study, we need to do twice as much damage control to achieve the same security as say, our colleague in the next office who is interested in a safer topic. Accepting controversy as par for the course for our fields or topics of interest can free up energy to get on with the job. Seeking peers or supervision to distinguish the roles of activist and researcher, how they are different and how they integrate in one's own particular circumstance, is important. Having a support system of other colleagues whom you feel supports you or who have faced similar challenges is vital. In addition, nurturing and protecting personal space - relationships, recreation, time outs, etc.-is critical for ensuring that personal health is maintained.
Each person will have her/his own reaction to a media event. Following a challenge, it is normal to feel anything from exhausted to traumatized to vengeful, left with selfdoubts or depressed feelings. No matter how supportive they were, it is common to be left feeling unsupported by team (e.g., staff, superiors, or institution). To avoid burn out, it is important to acknowledge such feelings and to address them appropriately (for example, by discussing them with a colleague(s) or friend). Affirming your strategies for handling conflict and seeking role models to teach you what strategies they use also can be helpful training for future incidents.

How to Handle Social Media during Controversial Situations

Social media are Internet-based applications that build on the ideological and technological foundations of Web 2.0 to allow the creation and exchange of User-Generated Content (Kaplan and Haenlien 2010). There are over 200 social media websites (Wikipedia.org 2012), and social networking now accounts for $22 \%$ of all time spent online in the USA (Nielsenwire 2010). Some of the more popular social media sites are: Facebook, Twitter, Flickr, Pinterest, YouTube, and Tumblr to name a few.

In April 2012, there were 845 million active users per month on Facebook, 175 million tweets a day on Twitter, 57 million members on LinkedIn, and 2 billion views per day on YouTube, while daily users of Pinterest have increased by more than 145\% since January 1, 2012 (Bullas 2012). As mentioned earlier in the protocol, the key to successful handling of controversial situations is careful and adequate planning. With such widespread and rapid sharing of information, it is essential to anticipate the role of social media when developing a written emergency protocol to manage controversial situations.

As it is usually not possible to stop the use of social media by those questioning your work, we deem this as beyond our control. Because part of any public relations protocol is to take measures to control the message, it is important to consider message control through social media with stakeholders and staff. With social media, posting to these sites is often tantamount to reporting to the media. Thus, it is essential to establish clear guidelines for all key personnel, staff, and affiliated stakeholders regarding uses of social media during a breaking news story. Just as only certain individuals affiliated with a study may speak directly to the media, only authorized individuals should post, share, or comment on the story. Furthermore, it is imperative that everyone recognize that these guidelines help the principal investigator to control and contain the message and ensure that the study team, the institution, and the funding agency present a united front and stay on message. 
One important reason to address social media explicitly with everyone associated with the research is that there is no consensus on what is or is not acceptable to share with a social network. During the MINTS media event, at least two staff members, acting in good faith and accustomed to posting updates on Facebook and Twitter about their day, shared what was happening with their friends, one or two of whom then shared it with their network. Fortunately, we caught it and were able to stop the information from being disseminated further, since we wanted to keep a low profile. However, the experience taught us that we need to be specifying precisely what is and is not allowed to be shared through social media. In particular, the principal investigator and team need to decide (1) what information about the study or media event, if any, can be shared electronically; and (2) what and to whom, information about a person's "day" is acceptable to share. Since needs may differ between team members, such as updating partners on changed plans or informing friends of the day's events, training everyone on boundaries is essential to a coordinated, consistent response.

In considering approaches to social media protocols, there are two extremes. One is a total blackout strategy, where the study attempts to avoid all media about a story and the other is a maximum disclosure and dissemination strategy where the study uses the event to disseminate publicity about the story.

\section{Considerations in a Blackout Strategy}

If the news story is an attack on the research (e.g., a petition to defund the study), we recommend the study team consider a complete blackout strategy when it comes to social media. By implementing such a strategy, the hope is that the news story will die, since engaging in social media (e.g., tweeting about the news story or posting it on a Facebook wall) runs the risk of prolonging the story. The four main reasons to adopt the blackout strategy are:

1. Blackouts reduce the opportunity for the story to appear in Twitter feeds which limits the new story's ability to gain traction and coverage on major news channels. News agencies often monitor Twitter for trending topics and decide to cover stories that generate a large amount of activity (e.g., re-tweets).

2. Blackouts reduce the likelihood of staff posting content or images that can be reposted by friends in their network and go viral. Once something is posted, it becomes impossible to control the message.

3. Blackouts reduce ambiguity among staff in terms of what is and is not allowed.

4. Blackouts are also a way to protect the team members since their name is less likely to be associated with the news story.
Considerations in a Media Maximum Dissemination Strategy

A team may wish to use the new story about the study to disseminate publicity about a health concern, intervention or study findings, and hence adopt a maximum dissemination strategy. Here, the hope is that the new story will provide the platform to inform the public. For such a scenario, a series of media releases coordinated with multiple Twitter postings (e.g., Facebook or Tumblr) will increase the likelihood of media attention on the study and on the findings of the study.

Dealing with Colleagues and Other People who are a Part of Your Social Media Network

It is always possible that someone in a staff member's network of friends will tweet, post, or otherwise ask about the news story. This should be brought to the attention of the principal investigator. The principal investigator, or a designee, should then prepare a short message to send to followers that can be posted on a blog, on Tumblr or on Twitter (not to exceed 140 characters). This could be something like: "We are aware of this news story and will be responding shortly."

\section{Monitoring Social Media}

While the media event is continuing, we recommend that at least one staff member be assigned exclusively, if possible, to monitor what is trending on social media and to provide daily updates to the investigators. The daily report should include the number of daily tweets, comments where the news story is posted, any blogging about the story, and Google searches, including text, images, and visual media searches. We recommend that this person prepare a spreadsheet that can be used to record all this information in an efficient manner. This becomes vital to gauge if the story is de-escalating or increasing in traction. After the situation has ended, we recommend that all media outlets be monitored monthly to make sure that the story is "dead".

\section{What if the Story Does Not "Die"?}

Both Twitter messages and media releases should be prepared and, where possible, approved by the principal investigator and public relations officials at the institution in case a story becomes prolonged and they need to be used. It is important to share the monitoring of social media data with key persons so that everyone is aware of the talking points to be put across in case the story does not "die". Additionally, the tweets prepared by the principal investigator should include a 
link to the "official message" to avoid being perceived as engaging in a "flame war" (i.e., a heated online argument based on ad hominem attacks).

Another option is to put together a website with relevant information in lay terms that people, including news outlets, can access to get accurate and unbiased information from your perspective.

\section{Intellectual Property Rights to Study Materials}

Both media and organizations questioning your research may post images or your materials on their website or a website (e.g., YouTube or Facebook). It is essential to ensure that the study has copyrights to all study materials (e.g., $\operatorname{logos}$, videos, curriculum, etc). If you find out that your materials have been posted without permission, we recommend that you work with your Counsel to issue a cease and desist. The organization that posted your materials has 60 days to take it down. Where copyrighted material has been used without permission on other sites (e.g., Twitter, YouTube), initiating a complaint usually results in it being taken down within $48 \mathrm{~h}$.

\section{Study Website and Electronic Media Management}

The principal investigator needs to have control of all online study-related media. This includes study website, study Facebook page, study blog, etc. By having full control, the principal investigator, or her/his designee, should be able to access the sites from any location and change content as needed during a story. Clearly, a study cannot be responsive if they need to wait hours or days to update their media pages. To protect all study staff and students, we recommend that the regular contact information (e.g., how to contact the project coordinator) be removed from the website during a media event, and replaced with whatever information is preferred for media. This prevents anyone from contacting staff directly and reduces the stress level of employees during such an event.

\section{Conclusion}

Perhaps in an ideal world, everyone would value scientific research and perceive it as a noble quest to advance human knowledge and health. In the real world, some research areas become politicized and/or controversial. Hence, we recommend studies consider a public relations emergency protocol. In the last decade, the explosion of social media has changed how we communicate and so, in addition to how to handle the press, it is equally important to anticipate how to address social media. Our experience has been that an emergency public relations protocol is needed, at least for all studies in controversial areas. It may even be good practice for noncontroversial studies as well. The exercise of developing a protocol, in our experience, strengthens a study, can help prevent or manage events which arise. Our hope in sharing this information is that other studies may benefit by using and adapting these protocols.

Acknowledgments The authors would like to acknowledge Dr. Deborah Rugg for her idea to formulate an emergency public relations protocol for controversial HIV prevention studies and Ms. Terri Charest for her contributions to earlier drafts of this manuscript. The examples in this protocol were selected from the Men's INTernet Study III (MINTS-III) for HIV prevention, an NIMH-funded online HIV prevention intervention study (2R01MH063688-09). We gratefully acknowledge the project coordinators of the HIV/STI Intervention \& Prevention Studies program for helping to implement this protocol on several studies. We thank Dr. Alex Iantaffi and Mr. Daniel Holsinger who advised on the manuscript in the area of social media and Dr. Michael Wilkerson, Mr. Jared Shenk, and Mr. Jeremy Grey for editorial suggestions and revisions.

\section{References}

Bullas, J. (2012). 48 Significant social media facts, figures and statistics plus 7 infographics. http://www.jeffbullas.com/2012/04/23/ 48-significant-social-media-facts-figures-and-statistics-plus-7infographics. Accessed 3 May 2012

Coombs, W. T. (2007). Crisis management and communications. http://www.instituteforpr.org/topics/crisis-management-andcommunications/. Accessed 29 April 2012.

Coombs, W. T., \& Holladay, S. J. (1996). Communication and attributions in a crisis: an experimental study of crisis communication. Journal of Public Relations Research, 8(4), 279-295.

Coombs, W. T., \& Holladay, S. J. (2001). An extended examination of the crisis situation: a fusion of the relational management and symbolic approaches. Journal of Public Relations Research, 13 (4), 321-340.

Covello, V. T. (2003). Best practices in public health risk and crisis communication. Journal of Health Communication: International Perspective, 8(1), 5-8.

Covello, V. T. (2012). Risk and crisis communication: communicating effectively in high concern, high stress, or low trust situations. http:// www.cdph.ca.gov/programs/cobbh/Documents/10\%20DR.\%20 VINCENT\%20COVELLO\%20a.Slides.San\%20Diego.pdf. Accessed 8 May 2012.

Covello, V. T., Sandman, P., \& Slovic, P. (1998). Risk communication, risk statistics and risk comparisons. Washington, D.C.: Chemical Manufacturers Association.

Kaplan, M. A., \& Haenlien, M. (2010). Users of the world, unite! The challenges and opportunities of social media. Business Horizons, $53,59-68$.

Kasperson, R., Renn, O., Slovic, P., \& Brown, H. (2006). The social amplification of risk: a conceptual framework. Risk Analysis, 8(2), 177-187.

Kelley, H. H., \& Michela, J. L. (1980). Attribution theory \& research. Annual Review of Psychology, 31, 457-501.

Marra, F. (1998). Crisis communication plans: poor predictors of excellent crisis public relations. Public Relations Review, 24(4), $461-474$. 
Nielsenwire. (2010, 8/2/2010). What Americans do online: Social media and games dominate activity. http://blog.nielsen.com/nielsenwire/ online_mobile/what-americans-do-online-social-media-and-gamesdominate-activity/. Accessed 4 May 2012

Oak Ridge Associated Universities (ORAU). (2012). Emergency/crisis/risk definitions. http:/www.orau.gov/cdcynergy/erc/content/ activeinformation/resources/EmergencyCrisisRiskCommunication Definitions.pdf. Accessed 7 May 2012
Reynolds, B., \& Seeger, M. (2005). Crisis and emergency risk communication as an integrative model. Journal of Health Communication: International Perspective, 10(1), 43-55.

Sandman, P. (1993). Responding to community outrage: strategies for effective risk communication. Virginia: AIHA.

Wikipedia.org. (2012). List of social networking sites. http:// en.wikipedia.org/wiki/List_of_social_networking_websites. Accessed 3 May 2012 